Research Article

\title{
Computation of the Eigenpairs of Two-Parameter Sturm-Liouville Problems Using the Regularized Sampling Method
}

\author{
B. Chanane and A. Boucherif \\ Department of Mathematics and Statistics, King Fahd University of Petroleum and Minerals, Dhahran 31261, Saudi Arabia \\ Correspondence should be addressed to B. Chanane; chanane@kfupm.edu.sa
}

Received 10 April 2014; Accepted 28 May 2014; Published 9 June 2014

Academic Editor: Juan J. Nieto

Copyright (c) 2014 B. Chanane and A. Boucherif. This is an open access article distributed under the Creative Commons Attribution License, which permits unrestricted use, distribution, and reproduction in any medium, provided the original work is properly cited.

This paper deals with the computation of the eigenvalues of two-parameter Sturm-Liouville (SL) problems using the Regularized Sampling Method, a method which has been effective in computing the eigenvalues of broad classes of SL problems (singular, nonself-adjoint, nonlocal, impulsive, etc.). We have shown, in this work that it can tackle two-parameter SL problems with equal ease. An example was provided to illustrate the effectiveness of the method.

\section{Introduction}

In an interesting paper published in 1963, Arscott [1] showed that the method of separation of variables used in solving boundary value problems for Laplace's equation leads to a two-parameter eigenvalue problem for ordinary differential equations with the auxiliary requirement that the solutions satisfy boundary conditions at several points. This has led to an extensive development of multiparameter spectral theory for linear operators (see, e.g., [2-8]). In [9], the authors give an overview of results on two-parameter eigenvalue problems for second order linear differential equations. Several properties of corresponding eigencurves are given. In [5], the authors have obtained interesting geometric properties of the eigencurves (e.g., the condition of transversal intersections is equivalent to the simplicity of the eigenvalues in the sense of Chow and Hale). All the above works are concerned with the theoretical aspect of the existence of eigenvalues. Also, several authors have dealt with the theoretical numerical analysis of two-parameter eigenvalue problems, $[10,11]$ and the references therein as well as the works of Plestenjak and his collaborators [12-15]. Eigenvalue problems have played a major role in the applied sciences. Consequently, the problem of computing eigenvalues of one-parameter problems has attracted many researchers (see, e.g., $[16,17]$ and the references therein).

Concerning the computations of eigenvalues of oneparameter Sturm-Liouville problems, the authors in [18] introduced a new method based on Shannon's sampling theory. It uses the analytic properties of the boundary function. The method has been generalized to a class of singular problems of Bessel type [19]. The books by Atkinson [20], Chow and Hale [21], Faierman [22], McGhee and Picard [23], Sleeman [24], Volkmer [25], and the long awaited monograph by Atkinson and Mingarelli [26] contain several results on eigenvalues of multiparameter Sturm-Liouville problems and the corresponding bifurcation problems. However, no attempt has been made to compute the eigenvalues of two-parameter Sturm-Liouville problems using the approach based on the Regularized Sampling Method introduced recently by the first author in [27] to compute the eigenvalues of general SturmLiouville problems and extended to the case of singular [28], non-self-adjoint [29], nonlocal [30], and impulsive SLPs [31]. We will consider, in this paper, the computation of the eigenpairs of two-parameter Sturm-Liouville problems with three-point boundary conditions using the Regularized Sampling Method. 


\section{The Characteristic Function}

Consider the two-parameter Sturm-Liouville problem as follows,

$$
\begin{array}{cc}
-y^{\prime \prime}+q y=\left(\mu_{1}^{2} w_{1}+\mu_{2}^{2} w_{2}\right) y, & 0<x<1, \\
y(0)=0, \quad y(c)=0, & y(1)=0,
\end{array}
$$

where $w_{1}$ and $w_{2}$ are positive and in $\mathscr{C}^{2}[0,1], q \in L[0,1]$, and $c \in(0,1)$ some given constant.

By an eigenvalue of (1) we mean a value of the couple $\left(\mu_{1}, \mu_{2}\right)$ for which problem (1) has a nontrivial solution. Conditions that insure the existence of eigenvalue are given in $[2-4,7,32,33]$. In fact, under fairly general conditions, it has been shown (see $[21,34]$ ) that there are smooth curves of eigenvalues (actually eigenpairs). Our objective is to effectively localize the eigencurves in the parameter $\left(\mu_{1}, \mu_{2}\right)$-plane. We should point out that we have restricted our attention to Dirichlet boundary conditions in order to eliminate technical details that might obscure the ideas.

We will associate with (1) the initial value problem as follows,

$$
\begin{gathered}
y^{\prime \prime}+\left(\mu_{1}^{2} w_{1}+\mu_{2}^{2} w_{2}\right) y=q y, \quad 0<x<1, \\
y(0)=0, \quad y^{\prime}(0)=1
\end{gathered}
$$

and deal first with the unperturbed case $(q=0)$ then with the perturbed case $(q \neq 0)$.

2.1. The Unperturbed Case $(q=0)$. In this case, $(2)$ reduces to

$$
\begin{array}{ll}
\varphi_{1}^{\prime \prime}+w \varphi_{1}=0, & 0<x<1, \\
\varphi_{1}(0)=0, & \varphi_{1}^{\prime}(0)=1,
\end{array}
$$

where $w=\mu_{1}^{2} w_{1}+\mu_{2}^{2} w_{2}$.

Theorem 1. The solution $\varphi_{1}$ of (3) is an entire function of $\left(\mu_{1}, \mu_{2}\right) \in \mathbb{C}^{2}$ for each fixed $x \in(0,1]$ of order $(1,1)$ and type $\left(\sigma_{1}(x), \sigma_{2}(x)\right)$ and satisfies the following estimate,

$$
\left|\varphi_{1}(x)\right| \leq K_{1} \exp \left[\sigma_{1}(x)\left|\mu_{1}\right|+\sigma_{2}(x)\left|\mu_{2}\right|\right], \quad\left(\mu_{1}, \mu_{2}\right) \in \mathbb{C}^{2}
$$

for each fixed $x \in(0,1]$, where $\sigma_{i}(x)=2\left\{x \int_{0}^{x} w_{i}(\xi) d \xi\right\}^{1 / 2}$ for $i=1,2$.

Proof. From (3), we get the following integral equation,

$$
\varphi_{1}(x)=x-\int_{0}^{x}(x-\xi)\left[\mu_{1}^{2} w_{1}(\xi)+\mu_{2}^{2} w_{2}(\xi)\right] \varphi_{1}(\xi) d \xi .
$$

Let

$$
\begin{gathered}
\varphi_{1,0}(x)=x \\
\varphi_{1, n+1}(x)=\int_{0}^{x}(x-\xi)\left[\mu_{1}^{2} w_{1}(\xi)+\mu_{2}^{2} w_{2}(\xi)\right] \varphi_{1, n}(\xi) d \xi
\end{gathered}
$$

We will show, by induction on $n$, that

$$
\begin{array}{r}
\left|\varphi_{1, n}(x)\right| \leq \frac{x}{n !(n+1) !}\left\{x \int_{0}^{x}\left[\left|\mu_{1}\right|^{2} w_{1}(\xi)+\left|\mu_{2}\right|^{2} w_{2}(\xi)\right] d \xi\right\}^{n}, \\
n \geq 0 .
\end{array}
$$

It is true for $n=0$. Assume it is true for $n$. We will show that it is true for $n+1$. Indeed, from (6), we have

$$
\begin{aligned}
& \left|\varphi_{1, n+1}(x)\right| \\
& \leq \int_{0}^{x}(x-\xi)\left[\left|\mu_{1}\right|^{2} w_{1}(\xi)+\left|\mu_{2}\right|^{2} w_{2}(\xi)\right] \\
& \quad \times \frac{\xi}{n !(n+1) !}\left\{\xi \int_{0}^{\xi}\left[\left|\mu_{1}\right|^{2} w_{1}(\tau)+\left|\mu_{2}\right|^{2} w_{2}(\tau)\right] d \tau\right\}^{n} d \xi .
\end{aligned}
$$

Using the fact that the expression $(x-\xi) \xi^{n+1}$ attains its maximum at $\xi=((n+1) /(n+2)) x$ over $[0, x]$, we get

$$
\begin{aligned}
& \left|\varphi_{1, n+1}(x)\right| \\
& \leq \frac{x}{n !(n+1) !} \frac{1}{n+1} \frac{1}{n+2}\left(\frac{n+1}{n+2} x\right)^{n+1} \\
& \quad \times\left\{\int_{0}^{x}\left[\left|\mu_{1}\right|^{2} w_{1}(\tau)+\left|\mu_{2}\right|^{2} w_{2}(\tau)\right] d \tau\right\}^{n+1}
\end{aligned}
$$

and using the fact that $(n+1) /(n+2)<1$, we obtain

$$
\begin{aligned}
\left|\varphi_{1, n+1}(x)\right| \leq & \frac{x}{(n+1) !(n+2) !} \\
& \times\left\{x \int_{0}^{x}\left[\left|\mu_{1}\right|^{2} w_{1}(\tau)+\left|\mu_{2}\right|^{2} w_{2}(\tau)\right] d \tau\right\}^{n+1}
\end{aligned}
$$

that is, (7) is true for $n+1$. Hence, it is true for all $n \geq 0$.

Now, $\varphi_{1}(x)=\sum_{n \geq 0}(-1)^{n} \varphi_{1, n}(x)$ and the series is absolutely and uniformly convergent since

$$
\begin{aligned}
\left|\varphi_{1}(x)\right|= & \left|\sum_{n \geq 0}(-1)^{n} \varphi_{1, n}(x)\right| \leq \sum_{n \geq 0}\left|\varphi_{1, n}(x)\right| \\
\leq & x \sum_{n \geq 0} \frac{1}{n !(n+1) !} \\
& \times\left\{x \int_{0}^{x}\left[\left|\mu_{1}\right|^{2} w_{1}(\tau)+\left|\mu_{2}\right|^{2} w_{2}(\tau)\right] d \tau\right\}^{n} \\
= & x I_{1}\left(\left\{2 \sqrt{\left.x \int_{0}^{x}\left[\left|\mu_{1}\right|^{2} w_{1}(\tau)+\left|\mu_{2}\right|^{2} w_{2}(\tau)\right] d \tau\right\}}\right)\right. \\
& \times\left\{x \int_{0}^{x}\left[\left|\mu_{1}\right|^{2} w_{1}(\tau)+\left|\mu_{2}\right|^{2} w_{2}(\tau)\right] d \tau\right\}^{-1 / 2},
\end{aligned}
$$


where $I_{1}$ is the modified Bessel function of the first kind order 1 as follows,

$$
I_{1}(z)=\sum_{n \geq 0} \frac{1}{n !(n+1) !}\left(\frac{z}{2}\right)^{2 n+1}
$$

Using the fact that $I_{1}(z) \sim e^{z} / \sqrt{2 \pi z}$ as $z \rightarrow \infty$, we get

$$
\begin{aligned}
& \left|\varphi_{1}(x)\right| \\
& \quad \leq K_{1} \exp \left[2\left\{x \int_{0}^{x}\left[\left|\mu_{1}\right|^{2} w_{1}(\tau)+\left|\mu_{2}\right|^{2} w_{2}(\tau)\right] d \tau\right\}^{1 / 2}\right] \\
& \quad \leq K_{1} \exp \left[\sigma_{1}(x)\left|\mu_{1}\right|+\sigma_{2}(x)\left|\mu_{2}\right|\right] .
\end{aligned}
$$

Therefore, $\varphi_{1}$ is an entire function of $\left(\mu_{1}, \mu_{2}\right) \in \mathbb{C}^{2}$, as a uniformly convergent series of entire functions, for each fixed $x \in(0,1]$, of order $(1,1)$ and type $\left(\sigma_{1}(x), \sigma_{2}(x)\right)$. This concludes the proof.

We will make use of Liouville-Green's transformation

$$
\begin{gathered}
t(x)=\int_{0}^{x} \sqrt{w(\xi)} d \xi \\
z(t)=\{w(x)\}^{1 / 4} \varphi_{1}(x)
\end{gathered}
$$

to bring (2) to the following form,

$$
\begin{gathered}
\frac{d^{2} z}{d t^{2}}+\{1+r(t)\} z=0, \quad 0<t<\int_{0}^{1} \sqrt{w(\xi)} d \xi \\
z(0)=0, \quad \frac{d z}{d t}(0)=\{w(0)\}^{-1 / 4},
\end{gathered}
$$

which can be written as an integral equation as follows,

$$
z(t)=\{w(0)\}^{-1 / 4} \sin t-\int_{0}^{t} \sin (t-\tau) r(\tau) z(\tau) d \tau,
$$

where $r(t)=\left.\left[\{w(x)\}^{-3 / 4}\left(d^{2} / d x^{2}\right)\{w(x)\}^{-1 / 4}\right]\right|_{x=x(t)}$.

Returning to the original variables, we deduce that $\varphi_{1}$ satisfies the following integral equation,

$$
\begin{aligned}
\varphi_{1}(x)= & \{w(0) w(x)\}^{-1 / 4} \sin \left\{\int_{0}^{x} \sqrt{w(\xi)} d \xi\right\} \\
& -\int_{0}^{x} \sin \left(\int_{\bar{x}}^{x} \sqrt{w(\xi)} d \xi\right) \psi(x, \bar{x}) \varphi_{1}(\bar{x}) d \bar{x}
\end{aligned}
$$

where

$$
\begin{aligned}
\psi(x, \bar{x})= & \{w(x)\}^{-1 / 4}\{w(\bar{x})\}^{-3 / 4} \\
\times & {\left[-\frac{1}{4} w^{\prime \prime}(\bar{x})\{w(\bar{x})\}^{-1 / 2}\right.} \\
& \left.+\frac{5}{16}\left\{w^{\prime}(\bar{x})\right\}^{2}\{w(\bar{x})\}^{-3 / 2}\right] .
\end{aligned}
$$

We will present next some estimates whose proofs are immediate and left to the reader.
Lemma 2. The function $\psi(x, \bar{x})$ satisfies the following estimate,

$$
\begin{aligned}
& |\psi(x, \bar{x})| \sim \frac{K_{2}}{\left|\mu_{1}\right|+\left|\mu_{2}\right|}, \\
& \text { as }\left|\mu_{1}\right|+\left|\mu_{2}\right| \longrightarrow \infty, \quad\left(\mu_{1}, \mu_{2}\right) \in \mathbb{R}^{2} .
\end{aligned}
$$

Lemma 3. The function $\alpha$ defined by

$$
\alpha(x)=\left(\sin c\left\{\sigma_{1}(x) \mu_{1}+\sigma_{2}(x) \mu_{2}\right\}\right)^{m},
$$

where $\sin c(z)=z^{-1} \sin z$ and $m$ is a positive integer, is an entire function of $\left(\mu_{1}, \mu_{2}\right) \in \mathbb{C}^{2}$ for each fixed $x \in(0,1]$ of $\operatorname{order}(1,1)$ and type $\left(\sigma_{1}(x), \sigma_{2}(x)\right)$. Furthermore, $\alpha$ satisfies the following estimate,

$$
|\alpha(1)| \sim \frac{K_{3}}{\left|\mu_{1}\right|+\left|\mu_{2}\right|}, \quad \text { as }\left|\mu_{1}\right|+\left|\mu_{2}\right| \longrightarrow \infty,\left(\mu_{1}, \mu_{2}\right) \in \mathbb{R}^{2} .
$$

Lemma 4. The function $\varphi_{1}$ satisfies the following estimate,

$\left|\varphi_{1}(1)\right| \sim \frac{K_{4}}{\left|\mu_{1}\right|+\left|\mu_{2}\right|}, \quad$ as $\left|\mu_{1}\right|+\left|\mu_{2}\right| \longrightarrow \infty,\left(\mu_{1}, \mu_{2}\right) \in \mathbb{R}^{2}$.

Combining the above results, we obtain the following theorem.

Theorem 5. The function $\alpha \varphi_{1}$ is an entire function of $\left(\mu_{1}, \mu_{2}\right) \in$ $\mathbb{C}^{2}$ for each fixed $x \in(0,1]$ of order $(1,1)$ and type $((m+$ 1) $\left.\sigma_{1}(x),(m+1) \sigma_{2}(x)\right)$ and satisfies the estimate

$$
\begin{aligned}
& \left|\alpha(x) \varphi_{1}(x)\right| \sim \frac{K(x)}{\left(\left|\mu_{1}\right|+\left|\mu_{2}\right|\right)^{m+1}}, \\
& \text { as }\left|\mu_{1}\right|+\left|\mu_{2}\right| \longrightarrow \infty, \quad\left(\mu_{1}, \mu_{2}\right) \in \mathbb{R}^{2},
\end{aligned}
$$

where $K$ depends on $x \in(0,1]$ but is independent of $\left(\mu_{1}, \mu_{2}\right)$.

Let $\mathrm{PW}_{\beta_{1}, \beta_{2}}$ denote the Paley-Wiener space as follows,

$$
\operatorname{PW}_{\beta_{1}, \beta_{2}}=\left\{\begin{array}{c}
h\left(z_{1}, z_{2}\right) \text { entire, }\left|h\left(z_{1}, z_{2}\right)\right| \leq C \exp \left\{\beta_{1}\left|z_{1}\right|+\beta_{2}\left|z_{2}\right|\right\}, \\
\iint_{-\infty}^{\infty}\left|h\left(z_{1}, z_{2}\right)\right|^{2} d z_{1} d z_{2}<\infty
\end{array}\right\} ;
$$

we have the following.

Theorem 6. $\alpha(x) \varphi_{1}(x)$, as a function of $\left(\mu_{1}, \mu_{2}\right)$, belongs to the Paley-Wiener space $P W_{\beta_{1}, \beta_{2}}$, where $\left(\beta_{1}, \beta_{2}\right)=((m+$ 1) $\left.\sigma_{1}(x),(m+1) \sigma_{2}(x)\right)$ for each fixed $x \in(0,1]$.

2.2. The Perturbed Case $(q \neq 0)$. Let $\varphi_{1}$ and $\varphi_{2}$ be two linearly independent solutions of $\varphi^{\prime \prime}+w \varphi=0$ satisfying $\varphi_{1}(0)=$ $\varphi_{2}^{\prime}(0)=0$ and $\varphi_{1}^{\prime}(0)=\varphi_{2}(0)=1$; then, the method of variation of parameters shows that (2) can be written as the integral equation as follows,

$$
y(x)=\varphi_{1}(x)+\int_{0}^{x} \Phi(x, \xi) q(\xi) y(\xi) d \xi
$$

where $\Phi(x, \xi)=\varphi_{1}(\xi) \varphi_{2}(x)-\varphi_{2}(\xi) \varphi_{1}(x)$. 
Here again, it is not hard to show that $y(x)$ is an entire function of $\left(\mu_{1}, \mu_{2}\right)$ for each $x \in(0,1]$, of order $(1,1)$ and type $\left(\sigma_{1}(x), \sigma_{2}(x)\right)$. Multiplication by $\alpha$ gives a function $\alpha(x) y(x)$ of $\left(\mu_{1}, \mu_{2}\right)$ in a Paley-Wiener space $\mathrm{PW}_{\beta_{1}, \beta_{2}}$ for each $x \in(0,1]$. More specifically, we have the following.

Theorem 7. The function $\tilde{y}$, defined by $\tilde{y}(x)=\alpha(x) y(x)$, belongs to $P W_{\beta_{1}, \beta_{2}}$, where $\left(\beta_{1}, \beta_{2}\right)=\left((m+1) \sigma_{1}(x),(m+\right.$ 1) $\left.\sigma_{2}(x)\right)$ as a function of $\left(\mu_{1}, \mu_{2}\right) \in \mathbb{C}$ for each $x \in(0,1]$, and satisfies the following estimate,

$$
\begin{aligned}
& |\alpha(x) y(x)| \sim \frac{K(x)}{\left(\left|\mu_{1}\right|+\left|\mu_{2}\right|\right)^{m+1}}, \\
& \text { as }\left|\mu_{1}\right|+\left|\mu_{2}\right| \longrightarrow \infty, \quad\left(\mu_{1}, \mu_{2}\right) \in \mathbb{R}^{2},
\end{aligned}
$$

where $K$ depends on $x \in(0,1]$ but is independent of $\left(\mu_{1}, \mu_{2}\right)$.

Proof. Since $\Phi_{x x}+w \Phi=0, \Phi(t, t)=0$, and $\Phi_{x}(t, t)=1$, we have

$$
\begin{aligned}
\Phi(x, t)= & \{w(t) w(x)\}^{-1 / 4} \sin \left(\int_{t}^{x} \sqrt{w(\xi)} d \xi\right) \\
& -\int_{t}^{x} \sin \left(\int_{\bar{x}}^{x} \sqrt{w(\xi)} d \xi\right) \psi(x, \bar{x}) \Phi(\bar{x}, t) d \bar{x}
\end{aligned}
$$

so that

$$
\begin{aligned}
& |\Phi(x, t)| \sim \frac{K_{4}}{\left|\mu_{1}\right|+\left|\mu_{2}\right|} \leq K_{5}, \\
& \text { as }\left|\mu_{1}\right|+\left|\mu_{2}\right| \longrightarrow \infty, \quad\left(\mu_{1}, \mu_{2}\right) \in \mathbb{R}^{2},
\end{aligned}
$$

from which we get, after using Gronwall's lemma [35] on (25) and the estimate for $\varphi_{1}$,

$$
\begin{aligned}
& |y(x)| \\
& \quad \leq K_{1} \exp \left[\sigma_{1}(x)\left|\mu_{1}\right|+\sigma_{2}(x)\left|\mu_{2}\right|\right] \exp \left\{K_{5} \int_{0}^{x}|q(t)| d t\right\}, \\
& |y(1)| \leq K_{7} \exp \left[\sigma_{1}(1)\left|\mu_{1}\right|+\sigma_{2}(1)\left|\mu_{2}\right|\right], \\
& \quad \text { as }\left|\mu_{1}\right|+\left|\mu_{2}\right| \longrightarrow \infty, \quad\left(\mu_{1}, \mu_{2}\right) \in \mathbb{R}^{2}, \\
& |\alpha(1) y(1)| \\
& \leq K_{8} \exp \left[(m+1) \sigma_{1}(1)\left|\mu_{1}\right|+(m+1) \sigma_{2}(1)\left|\mu_{2}\right|\right], \\
& \quad \text { as }\left|\mu_{1}\right|+\left|\mu_{2}\right| \longrightarrow \infty, \quad\left(\mu_{1}, \mu_{2}\right) \in \mathbb{R}^{2} .
\end{aligned}
$$

Furthermore, we have

$$
\begin{aligned}
& |\alpha(x) y(x)| \sim \frac{K(x)}{\left(\left|\mu_{1}\right|+\left|\mu_{2}\right|\right)^{m+1}}, \\
& \text { as }\left|\mu_{1}\right|+\left|\mu_{2}\right| \longrightarrow \infty, \quad\left(\mu_{1}, \mu_{2}\right) \in \mathbb{R}^{2},
\end{aligned}
$$

where $K$ depends on $x \in(0,1]$ but is independent of $\left(\mu_{1}, \mu_{2}\right)$.
To summarize, in both cases, unperturbed and perturbed, the transform $\tilde{y}\left(x ; \mu_{1}, \mu_{2}\right)$ of the solution $y\left(x ; \mu_{1}, \mu_{2}\right)$ of $(2)$ is in a Paley-Wiener space $\mathrm{PW}_{\beta_{1}, \beta_{2}}$, where $\left(\beta_{1}, \beta_{2}\right)=((m+$ 1) $\left.\sigma_{1}(x),(m+1) \sigma_{2}(x)\right)$. Thus $\tilde{y}\left(x ; \mu_{1}, \mu_{2}\right)$ can be recovered at each $x \in(0,1]$ from its samples at the lattice points $\left(\mu_{1 j}, \mu_{2 k}\right)=\left(j\left(\pi /(m+1) \sigma_{1}(x)\right), k\left(\pi /(m+1) \sigma_{2}(x)\right)\right),(j, k) \in$ $\mathbb{Z}^{2}$ using the rectangular cardinal series $[36,37]$.

Theorem 8. Let $f \in P W_{\beta_{1}, \beta_{2}}$; then,

$$
\begin{aligned}
& f\left(\mu_{1}, \mu_{2}\right) \\
& =\sum_{j=-\infty}^{\infty} \sum_{k=-\infty}^{\infty} f\left(\mu_{1 j}, \mu_{2 k}\right) \\
& \quad \times \frac{\sin \beta_{1}\left(\mu_{1}-\mu_{1 j}\right)}{\beta_{1}\left(\mu_{1}-\mu_{1 j}\right)} \frac{\sin \beta_{2}\left(\mu_{2}-\mu_{2 k}\right)}{\beta_{2}\left(\mu_{2}-\mu_{2 k}\right)}
\end{aligned}
$$

with the convergence of the series being uniform and in $L_{d \mu_{1} d \mu_{2}}^{2}\left(\mathbb{R}^{2}\right)$, and $\mu_{m n}=n \pi / \beta_{m}, m=1,2, n \in \mathbb{Z}$.

Let $\sigma_{11}=\sigma_{1}(1), \sigma_{21}=\sigma_{2}(1), \sigma_{12}=\sigma_{1}(c)$, and $\sigma_{22}=\sigma_{2}(c)$.

The eigenpairs are therefore $\left(\mu_{1}^{2}, \mu_{2}^{2}\right)$, where $\left(\mu_{1}, \mu_{2}\right)$ solve the nonlinear system as follows,

$$
\begin{aligned}
& B_{1}\left(\mu_{1}, \mu_{2}\right)=0, \\
& B_{2}\left(\mu_{1}, \mu_{2}\right)=0,
\end{aligned}
$$

where

$$
\begin{aligned}
& B_{1}\left(\mu_{1}, \mu_{2}\right) \\
& \triangleq \frac{1}{\alpha(1)} \sum_{j=-\infty}^{\infty} \sum_{k=-\infty}^{\infty} \tilde{y}\left(1 ; \mu_{1 j}, \mu_{2 k}\right) \\
& \times \frac{\sin 2(m+1) \sigma_{11}\left(\mu_{1}-\mu_{1 j}\right)}{2(m+1) \sigma_{11}\left(\mu_{1}-\mu_{1 j}\right)} \\
& \times \frac{\sin 2(m+1) \sigma_{21}\left(\mu_{2}-\mu_{2 k}\right)}{2(m+1) \sigma_{21}\left(\mu_{2}-\mu_{2 k}\right)}, \\
& B_{1}\left(\mu_{1}, \mu_{2}\right) \sum_{1}^{\alpha(c)} \sum_{j=-\infty}^{\infty} \sum_{k=-\infty}^{\infty} \tilde{y}\left(c ; \mu_{1 j}, \mu_{2 k}\right) \\
& \times \frac{\sin 2(m+1) \sigma_{12}\left(\mu_{1}-\mu_{1 j}\right)}{2(m+1) \sigma_{12}\left(\mu_{1}-\mu_{1 j}\right)} \\
& \times \frac{\sin 2(m+1) \sigma_{22}\left(\mu_{2}-\mu_{2 k}\right)}{2(m+1) \sigma_{22}\left(\mu_{2}-\mu_{2 k}\right)} .
\end{aligned}
$$


TABLE 1

\begin{tabular}{lccc}
\hline$\mu_{1}$ (exact) & $\mu_{2}$ (exact) & $\mu_{1}(\mathrm{RSM})$ & $\mu_{2}(\mathrm{RSM})$ \\
\hline 7.788149097670813 & 7.5908224365786845 & 7.78814883048352 & 7.590823605399021 \\
4.194056047341936 & 22.273796542861913 & 4.194057357011064 & 22.273795699572364 \\
15.597607295800684 & 15.165889997583099 & 15.597605904429917 & 15.1658929384375 \\
13.87615754699624 & 30.597837626955204 & 13.876157718920586 & 30.59783713797321 \\
23.40242244972004 & 22.744341450749697 & 23.40242640980619 & 22.744329536815076 \\
9.51130982713563 & 44.296491367611544 & 9.511333341150682 & 44.29647446214639 \\
39.31833491621106 & 15.670248767892955 & 39.31833152483279 & 15.670257662031721 \\
47.18632817417246 & 24.620489734469363 & 47.186345272524804 & 24.62038504864854 \\
46.81208951678993 & 45.483251580157955 & 46.813097550330646 & 45.480149444567346 \\
30.03437464767369 & 46.558148101315844 & 30.034392317076748 & 46.55811263809656 \\
\hline
\end{tabular}

\section{A Numerical Example}

We will consider in this section the two-parameter SturmLiouville problem with three-point boundary conditions given by

$$
\begin{gathered}
-y^{\prime \prime}=\left(\mu_{1}^{2}+\mu_{2}^{2} x\right) y, \quad 0<x<1, \\
y(0)=y(0.7)=y(1) .
\end{gathered}
$$

The general solution $y$ of the first differential equation can be expressed in terms of $\mathrm{Ai}$ and Bi functions [38] and their first derivatives as

$$
\begin{gathered}
y\left(x ; \mu_{1}, \mu_{2}\right) \\
=(-1)^{2 / 3}\left(\operatorname{Ai}\left(\frac{\sqrt[3]{-1} \mu_{1}^{2}}{\mu_{2}^{4 / 3}}\right) \operatorname{Bi}\left(\frac{\sqrt[3]{-1}\left(\mu_{1}^{2}+\mu_{2}^{2} x\right)}{\mu_{2}^{4 / 3}}\right)\right. \\
\left.-\operatorname{Ai}\left(\frac{\sqrt[3]{-1}\left(\mu_{1}^{2}+\mu_{2}^{2} x\right)}{\mu_{2}^{4 / 3}}\right) \operatorname{Bi}\left(\frac{\sqrt[3]{-1} \mu_{1}^{2}}{\mu_{2}^{4 / 3}}\right)\right) \\
\times\left(\mu _ { 2 } ^ { 2 / 3 } \left(\operatorname{Ai}^{\prime}\left(\frac{\sqrt[3]{-1} \mu_{1}^{2}}{\mu_{2}^{4 / 3}}\right) \operatorname{Bi}\left(\frac{\sqrt[3]{-1} \mu_{1}^{2}}{\mu_{2}^{4 / 3}}\right)\right.\right. \\
\left.\left.-\operatorname{Ai}\left(\frac{\sqrt[3]{-1} \mu_{1}^{2}}{\mu_{2}^{4 / 3}}\right) \mathrm{Bi}^{\prime}\left(\frac{\sqrt[3]{-1} \mu_{1}^{2}}{\mu_{2}^{4 / 3}}\right)\right)\right)^{-1} .
\end{gathered}
$$

Thus the eigenpairs $\left(\mu_{1}^{2}, \mu_{2}^{2}\right)$ can be obtained from the solutions $\left(\mu_{1}, \mu_{2}\right)$ of the following system,

$$
\begin{aligned}
& \tilde{y}\left(1 ; \mu_{1}, \mu_{2}\right)=0, \\
& \tilde{y}\left(c ; \mu_{1}, \mu_{2}\right)=0 .
\end{aligned}
$$

For numerical purposes, we have truncated the associated series to $|j|,|k| \leq N=50$ and took $m=5$ in the function $\alpha$. Thus, the approximate eigenpairs are seen as solutions of the following system,

$$
\begin{aligned}
& \tilde{y}^{[N]}\left(1 ; \mu_{1}, \mu_{2}\right)=0, \\
& \tilde{y}^{[N\}}\left(c ; \mu_{1}, \mu_{2}\right)=0 .
\end{aligned}
$$

Table 1 shows the exact eigenpairs together with their approximations using the Regularized Sampling Method (RSM).

\section{Conclusion}

In this paper, we have successfully computed the eigenpairs of two-parameter Sturm-Liouville problems using the regularized sampling method, a method which has been very efficient in computing the eigenvalues of broad classes of Sturm-Liouville problems (singular, non-self-adjoint, nonlocal, impulsive, etc.). We have shown in this work that it can tackle two-parameter SL problems with equal ease. An example was provided to illustrate the effectiveness of the method.

\section{Conflict of Interests}

The authors declare that there is no conflict of interests regarding the publication of this paper.

\section{Acknowledgment}

The authors are grateful to King Fahd University of Petroleum and Minerals for its usual support.

\section{References}

[1] F. M. Arscott, "Paraboloidal co-ordinates and Laplace's equation," Proceedings of the Royal Society of Edinburgh, vol. 66, pp. 129-139, 1963.

[2] F. M. Arscott, "Two-parameter eigenvalue problems in differential equations," Proceedings of the London Mathematical Society, vol. 14, pp. 459-470, 1964.

[3] P. Binding, "Perturbation and bifurcation of nonsingular multiparametric eigenvalues," Nonlinear Analysis: Theory, Methods \& Applications, vol. 8, no. 4, pp. 335-352, 1984.

[4] P. Binding, "Indefinite Sturm-Liouville theory via examples of two-parameter eigencurves," in Proceedings of the 1990 Dundee Conference on Differential Equations, R. Jarvis and B. D. Sleeman, Eds., pp. 38-49, Pitman, 1991.

[5] P. Binding and P. J. Browne, "Eigencurves for two-parameter selfadjoint ordinary differential equations of even order," Journal of Differential Equations, vol. 79, no. 2, pp. 289-303, 1989. 
[6] P. Binding and H. Volkmer, "Eigencurves for two-parameter Sturm-Liouville equations," SIAM Review, vol. 38, no. 1, pp. 2748, 1996.

[7] A. Boucherif, "Nonlinear three-point boundary value problems," Journal of Mathematical Analysis and Applications, vol. 77, no. 2, pp. 577-600, 1980.

[8] P. J. Browne and B. D. Sleeman, "A note on the characterisation of eigencurves for certain two parameter eigenvalue problems in ordinary differential equations," Glasgow Mathematical Journal, vol. 35, no. 1, pp. 63-67, 1993.

[9] P. Binding and P. J. Browne, "Spectral properties of twoparameter eigenvalue problems," Proceedings of the Royal Society of Edinburgh, vol. 89, no. 1-2, pp. 157-173, 1981.

[10] P. B. Bailey, "The automatic solution of two-parameter SturmLiouville eigenvalue problems in ordinary differential equations," Applied Mathematics and Computation, vol. 8, no. 4, pp. 251-259, 1981.

[11] E. K. Blum and A. R. Curtis, "A convergent gradient method for matrix eigenvector-eigentuple problems," Numerische Mathematik, vol. 31, no. 3, pp. 247-263, 1978/79.

[12] B. Plestenjak, "A continuation method for a right definite two-parameter eigenvalue problem," SIAM Journal on Matrix Analysis and Applications, vol. 21, no. 4, pp. 1163-1184, 2000.

[13] B. Plestenjak, "A continuation method for a weakly elliptic two-parameter eigenvalue problem," IMA Journal of Numerical Analysis, vol. 21, no. 1, pp. 199-216, 2001.

[14] M. E. Hochstenbach and B. Plestenjak, "A Jacobi-Davidson type method for a right definite two-parameter eigenvalue problem," SIAM Journal on Matrix Analysis and Applications, vol. 24, no. 2, pp. 392-410, 2002.

[15] M. E. Hochstenbach, T. Košir, and B. Plestenjak, "A JacobiDavidson type method for the two-parameter eigenvalue problem," SIAM Journal on Matrix Analysis and Applications, vol. 26, no. 2, pp. 477-497, 2004/05.

[16] P. B. Bailey, W. N. Everitt, and A. Zettl, "Computing eigenvalues of singular Sturm-Liouville problems," Results in Mathematics, vol. 20, no. 1-2, pp. 391-423, 1991.

[17] B. Chanane, "High order approximations of the eigenvalues of regular Sturm-Liouville problems," Journal of Mathematical Analysis and Applications, vol. 226, no. 1, pp. 121-129, 1998.

[18] A. Boumenir and B. Chanane, "Eigenvalues of S-L systems using sampling theory," Applicable Analysis, vol. 62, no. 3-4, pp. 323334, 1996.

[19] A. Boumenir and B. Chanane, "Computing eigenvalues of Sturm-Liouville systems of Bessel type," Proceedings of the Edinburgh Mathematical Society, vol. 42, no. 2, pp. 257-265, 1999.

[20] F. V. Atkinson, Multiparameter Eigenvalue Problems, Academic Press, New York, NY, USA, 1972.

[21] S. N. Chow and J. K. Hale, Methods of Bifurcation Theory, vol. 251, Springer, New York, NY, USA, 1982.

[22] M. Faierman, Two-Parameter Eigenvalue Problems in Ordinary Differential Equations, vol. 205 of Pitman Research Notes in Mathematics Series, Longman, Harlow, UK, 1991.

[23] D. McGhee and R. Picard, Cordes' Two-Parameter Spectral Representation Theory, Pitman Research Notes, Longman, Harlow, UK, 1988.

[24] B. D. Sleeman, Multiparameter Spectral Theory in Hilbert Space, Pitman Research Notes, Longman, Harlow, UK.

[25] H. Volkmer, Multiparameter Eigenvalue Problems and Expansion Theorems, vol. 1356 of Lecture Notes in Mathematics, Springer, Berlin, Germany, 1988.
[26] F. V. Atkinson and A. B. Mingarelli, Multiparameter Problems Sturm-Liouville Theory, CRC Press, Boca Raton, Fla, USA, 2011.

[27] B. Chanane, "Computation of the eigenvalues of SturmLiouville problems with parameter dependent boundary conditions using the regularized sampling method," Mathematics of Computation, vol. 74, no. 252, pp. 1793-1801, 2005.

[28] B. Chanane, "Computing the eigenvalues of singular SturmLiouville problems using the regularized sampling method," Applied Mathematics and Computation, vol. 184, no. 2, pp. 972978, 2007.

[29] B. Chanane, "Computing the spectrum of non-self-adjoint Sturm-Liouville problems with parameter-dependent boundary conditions," Journal of Computational and Applied Mathematics, vol. 206, no. 1, pp. 229-237, 2007.

[30] B. Chanane, "Computing the eigenvalues of a class of nonlocal Sturm-Liouville problems," Mathematical and Computer Modelling, vol. 50, no. 1-2, pp. 225-232, 2009.

[31] B. Chanane, "Sturm-Liouville problems with impulse effects," Applied Mathematics and Computation, vol. 190, no. 1, pp. 610626, 2007.

[32] M. Greguš, F. Neuman, and F. M. Arscott, “Three-point boundary value problems in differential equations," Journal of the London Mathematical Society, vol. 3, pp. 429-436, 1971.

[33] L. Turyn, "Sturm-Liouville problems with several parameters," Journal of Differential Equations, vol. 38, no. 2, pp. 239-259, 1980.

[34] A. Boucherif and N. Boukli-Hacene, "Two-parameter eigenvalue problems with time-dependent three-point boundary conditions," Maghreb Mathematical Review, vol. 8, no. 1-2, pp. 57-66, 1999.

[35] E. A. Coddington and N. Levinson, Theory of Ordinary Differential Equations, McGraw-Hill, New York, NY, USA, 1955.

[36] A. I. Zayed, Advances in Shannon's Sampling Theory, CRC Press, Boca Raton, Fla, USA, 1993.

[37] A. I. Zayed, "A sampling theorem for signals bandlimited to a general domain in several dimensions," Journal of Mathematical Analysis and Applications, vol. 187, no. 1, pp. 196-211, 1994.

[38] M. Abramowitz and I. A. Stegun, Handbook of Mathematical Functions with Formulas, Graphs, and Mathematical Tables, Dover Publications, New York, NY, USA, 1972. 


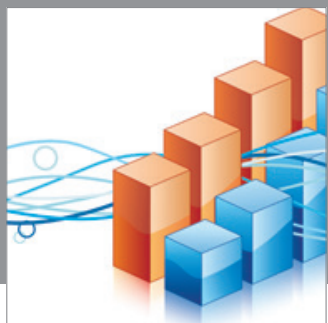

Advances in

Operations Research

mansans

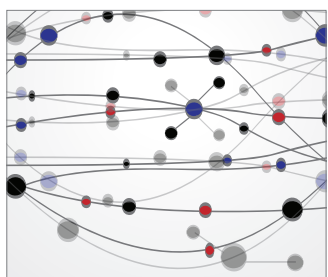

The Scientific World Journal
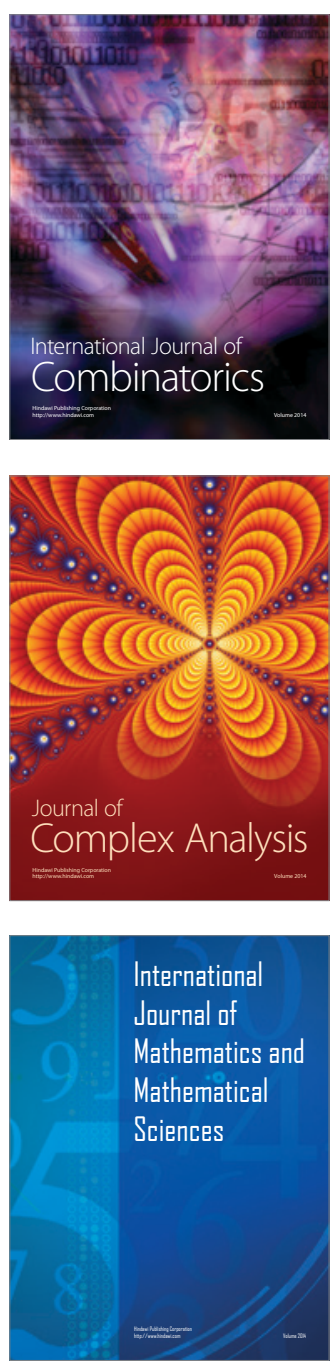
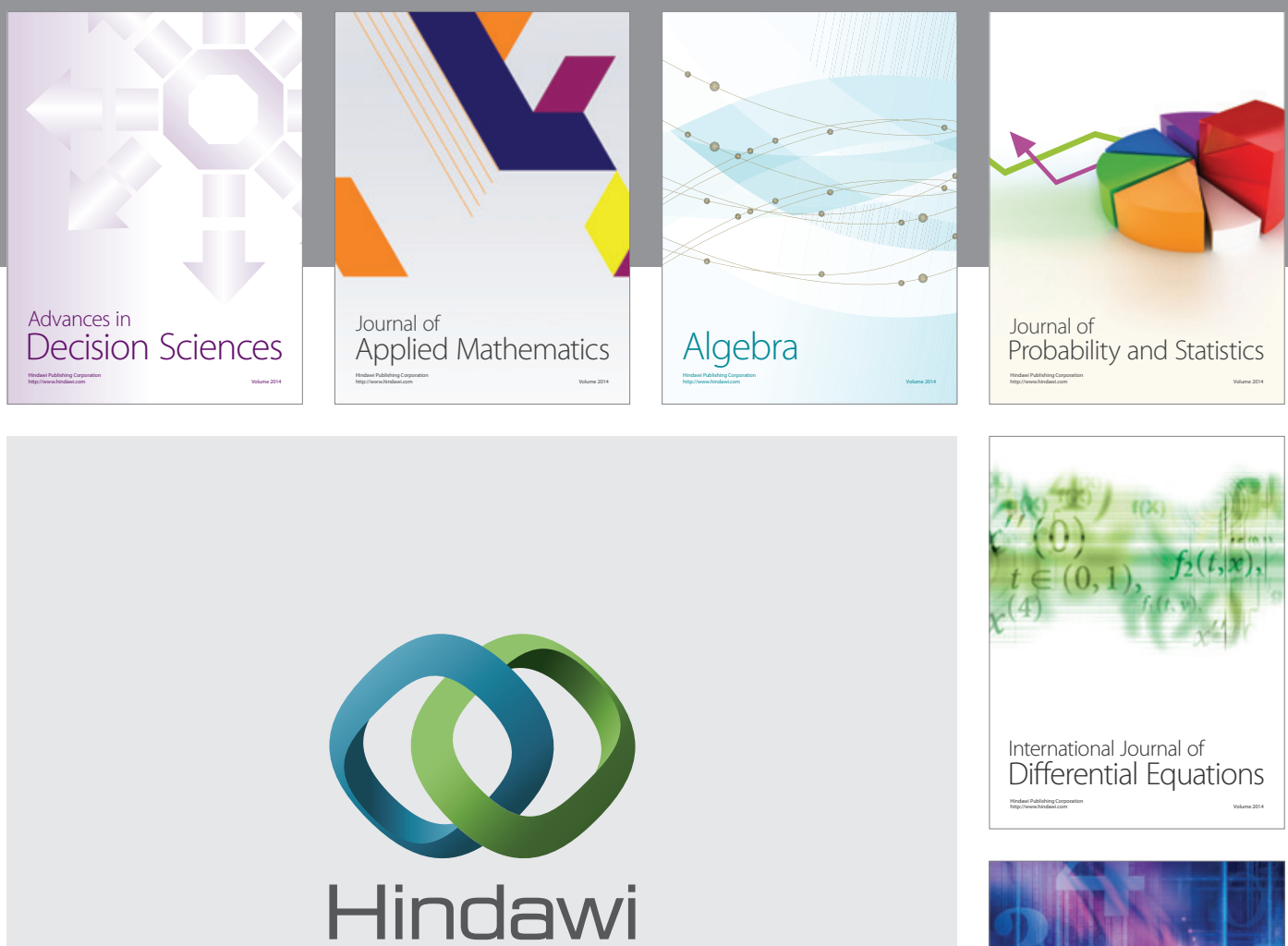

Submit your manuscripts at http://www.hindawi.com
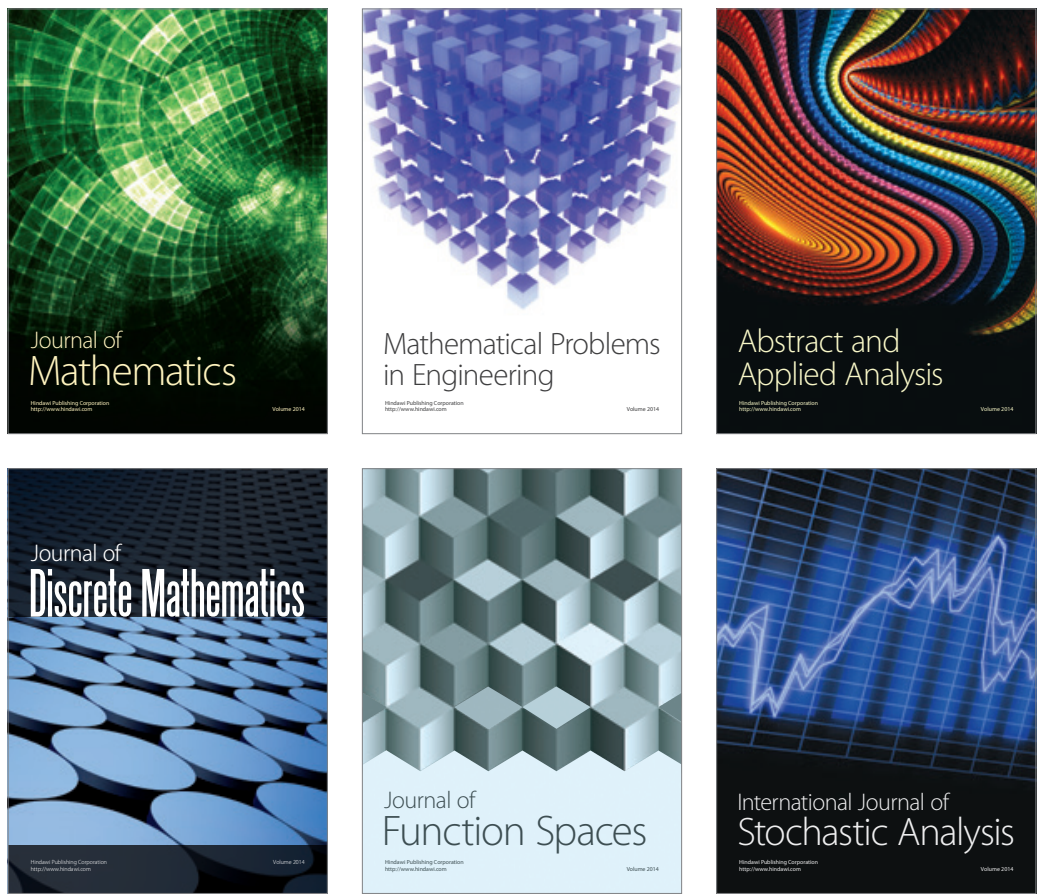

Journal of

Function Spaces

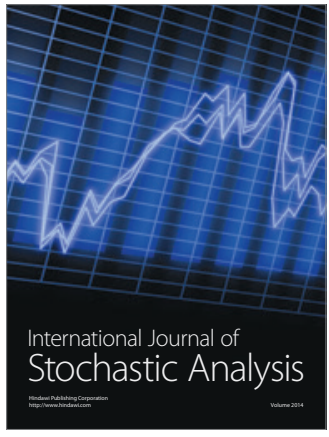

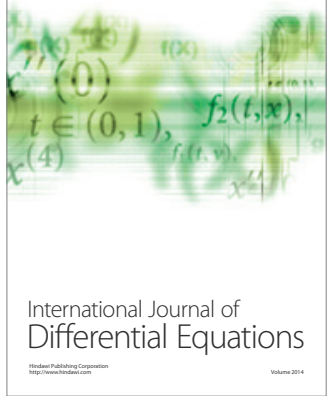
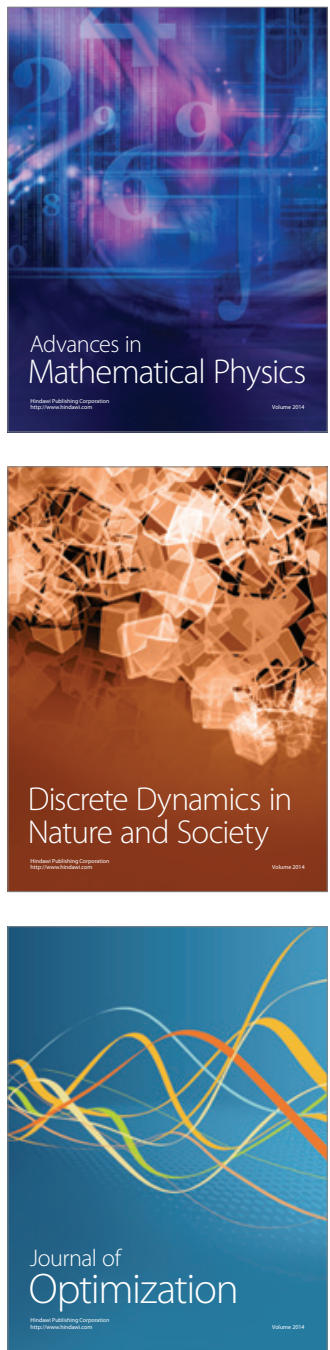